\title{
COMPRESSIBLE FLOW WITH VACUUM AND PHYSICAL SINGULARITY*
}

\author{
TAI-PING LIU' AND TONG YANG Y $^{\dagger}$
}

\begin{abstract}
In this paper, we will study the compressible flow with vacuum when the physical sigularity is prescribed at the vacuum boundary. The physical singularity at the boundary corresponds to the non-zero pressure effect on the evolution of the boundary and is the canonical behavior of the solution after finite waiting time. The local existence of solutions to both the systems of Euler equations with damping and the Euler-Poisson equations for gaseous stars are obtained by a coordinate transformation capturing this singular behavior at the vacuum. The local existence theorems with the physical boundary behaviour are new. The global existence and large time behavior are left to the future.
\end{abstract}

1. Introduction. The study of compressible flow with physical vacuum boundary behaviour has been a basic key open problem of the subject. The main difficulty comes from coincidence of the characteristics at the vacuum boundary, which results in the singular behaviour of the gas flow near the vacuum. There are local existence theories when the singularity near vacuum is assumed to be mild. On the other hand, such a mild singularity can not last and a stronger, physical singularity will developein finite time. This is known to occur naturally, for instance, in the evolution of the gaseous stars. The degeneracy of the Euler equations with the physical singularity prevents the application of the classical local existence theory for symmetric hyperbolic systems. In this paper, we resolve this problem and illustrate our ideas for two models: the Euler equations for isentropic compressible flow with damping and the Euler-Poisson equations for gaseous stars. Local existence of solutions with physical singularity will be proved by using a coordinate transformation, which precisely captures the singularity at the vacuum boundary. Similar analysis would apply to other physical systems. The global existence and large time behavior of solutions with the physical boundary is not in the scope of this paper and will be persued by authors in the future.

Since the Euler equations for isentropic flow with vacuum fails to be strictly hyperbolic at the vacuum states, the classical analysis of strictly hyperbolic equations is not applicable to the present situation. In fact, most of the main problems on this system are still open. For instance, it would be interesting to study the singular evolution of the interface, that is, to give a detailed description of how the solution near the interface changes and how it becomes from being initally smooth to the canonical behavior after finite time. Another would be the study of the nonlinear stability of the travelling wave solutions and the class of special solutions corresponding to the Barenblatt's solutions for the porous media equation obtained in [11]. It will be also interesting and important to give the time asymptotic equivalence between the Euler equations with damping and the porous media equation when vacuum occurs. Such studies would help us to understand the singularity, evolution of vacuum boundary and other rich phenomena caused by the vacuum. It would also help the design of the numerical schemes for the computation of the evolution of the vacuum boundary.

\footnotetext{
${ }^{*}$ Received January 6, 2000.

†Department of Mathematics, Stanford University. The research was supported in part by NSF Grant DMS-9803323.

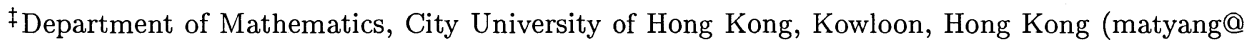
math.cityu.edu.hk). The research was supported in part by the RGC Competitive Earmarked Research Grant 9040468.
} 
Consider the one dimensional compressible Euler equations for isentropic flow with damping in Eulerian coordinates

$$
\begin{gathered}
\rho_{t}+(\rho u)_{x}=0 \\
\rho u_{t}+\rho u u_{x}+p(\rho)_{x}=-k \rho u
\end{gathered}
$$

where $\rho, u$ and $p(\rho)$ are density, velocity and pressure respectively. And the constant $k>0$ is the frictional coefficient. When the initial density function has compact support, the vacuum boundary $\Gamma$ is defined as

$$
\Gamma=\operatorname{cl}\{(\vec{x}, t) \mid \rho(\vec{x}, t)>0\} \cap \operatorname{cl}\{(\vec{x}, t) \mid \rho(\vec{x}, t)=0\} .
$$

The main difficulty caused by vacuum is that the system becomes degenerate, that is, characteristics coincide. They become zero in the Lagrangian coordinates. Therefore, even though the system is symmetrizable, in general the coefficients do not satisfy the usual local existence theories.

For Euler equations without damping and vacuum, the author in [22] gave a sufficient condition for the blowup of $C^{1}$ solutions. The nonexistence of $C^{1}$ solutions in [22] is related to the shock formation. However, the non-existence of global smooth solutions with vacuum is not so much related to the shock formation, but more to the developement of singularity at the vacuum. We therefore, in the following discussion, concentrate on the solutions containing no shocks.

When there is no frictional damping, it was shown that the shock waves vanish at the vacuum and the singular, non-shock behavior at the vacuum is similar to that of the centered rarefaction waves, [14]. Under some special condition on the initial velocity, the authors in [7] give the existence of global smooth solutions of Euler equations for an isentropic perfect gas in the $d$-dimensional Euclidean space.

When there is linear frictional damping and the solution is away from vacuum, the dissipative effect of damping prevents shock from forming. Since no other singularity emerges in the flow, it was shown that the system is time-asymptotically equivalent to the porous media equation, [8]. When the system contains both linear damping and vacuum, in [11], the author constructs a class of spherical symmetric solutions which converge to the self-similar solutions of the porous media equation,

$$
\begin{gathered}
k \rho_{t}=p(\rho)_{x x} \\
p(\rho)_{x}=-k \rho u
\end{gathered}
$$

So far this kind of equivalence with vacuum was only proved for this class of special solutions. It is also conjectured in [11] that the general behavior of the solution near $\Gamma$ satisfying

$$
0<\left|\frac{d c^{2}}{d x}\right|<\infty
$$

in the Eulerian coordinates after finite time, where $c=\sqrt{\frac{d}{d \rho} p(\rho)}$ is the sound speed.

A vacuum boundary is called physical if (1.2) is satisfied in a small neighborhood of the boundary.

Notice that this singular behavior implies that the pressure has a nonzero bounded effect on the evolution of the vacuum boundary. This can be seen from 
the equation for the evolution of the vacuum boundary which is given by the second equation of (1.1) as

$$
u_{t}+u u_{x}+\frac{1}{\gamma-1} c_{x}^{2}=-k u
$$

if the solution connects to vacuum continuously. When $c_{x}^{2}=0$ at the vacuum boundary, (1.3) means that the evolution of the vacuum boundary is independent of the gas inside the region so that it can not hold for all time $t$. Under the condition (1.2), the vacuum boundary moves according to the pressure gradient built near the boundary when time becomes large.

This also implies that if initial data $(\rho, u)(x, 0)$ are smooth, then there is a waiting time, before which the boundary does not move. After that time, the boundary will move due to the effect of the pressure. In [11], a class of travelling wave solutions with infinite total mass is also constructed. To our knowledge, these two families of solutions are the only non-trivial global solutions to the Euler equations with vacuum and damping where the vacuum boundary is clearly presented.

Along this direction, the authors in [13] show that the regular solutions can not be global if the density function has compact support.

The local existence of solutions with

$$
\frac{d c^{\alpha}}{d x}, \quad 0<\alpha \leq 1
$$

bounded away from zero across $\Gamma$ is also proved. It is noticed that this kind of phenomenon exists and $\alpha$ remains the same locally in time. When the initial data connect to vacuum states discontinuously, local existence for Euler equations without damping was proved in [12] by a polygonal method introduced in [5] for scalar equations and generalized in [10] for $p$-system.

When inf $\rho_{0}(\vec{x})=0$, the author in [18] proved the nonglobal existence of regular solutions by assuming that the initial data $\left(\rho_{0}(\vec{x}), \vec{u}_{0}(\vec{x})\right)$ have compact support, where $\vec{u}_{0}(\vec{x})$ is the initial velocity. For the Euler-Poisson equations governing gaseous stars, the authors in [16] proved the nonglobal existence of tame solutions under the condition of spherical symmetry. This nonglobal existence result can be generalized to the boundary condition $\frac{d}{d x} c^{2}=0$ instead of $\frac{d}{d x} c=0$ at vacuum. Local existence of tame or regular solutions for these two systems was proved in [15, 17] by using the symmetrization and the fixed point theorem. Solutions thus obtained correspond to those of $0<\alpha<\frac{2}{3}$ or $\alpha=1$ if the solutions are in the space $H^{2}$. Therefore, it does not include the stationary solution which can be explicitely described under the spherical symmetry condition. Using the same symmetrization, the author in [6] uses the paradifferential calculus of J.M. Bony, and the author in [3] uses the Gagliardo-Nirenberg inequality and Littlewood-Paley theory to obtain local existence of solutions without assuming the support of the initial density being compact. And these two results include the stationary solutions only when $\gamma=\frac{6}{5}$. Here the pressure function is assumed to satisfy the $\gamma$-law for polytropic gas, i.e., $p(\rho)=\sigma^{2} \rho^{\gamma}$.

Notice that the regular solutions defined in [13] is different from that in [17], where $\rho^{\gamma-1} \in C^{1}\left([0, T) \times \mathbf{R}^{3}\right)$ instead of $\rho^{\frac{\gamma-1}{2}} \in C^{1}\left([0, T) \times \mathbf{R}^{3}\right)$ is required. Since $c=\sigma \sqrt{\gamma} \rho^{\frac{\gamma-1}{2}}$ is a continuous function, the regular solution defined in [13] is more general than the one in [17], and thus the nonexistence theorem in [13] generalizes that of [17]. So far the proofs for the non-existence of global regular solutions either for invisid models given above or for the viscous models, cf. [13, 15, 16, 18, 23] etc., 
are based on the analysis of the support of the density. As shown by the special class of global solutions obtained in [11], the support of the density there is infinite. Therefore, deeper understanding on the development of the singularity at the vacuum is desired.

According to the above argument, the general behavior of the solutions near vacuum should be the one corresponding to $\alpha=2$. By introducing a coordinate transformation, we will prove the local existence theorem for this case in Section 2. Notice that this local existence theorem holds also for the situation without damping. However, it is well-known that the large time behavior of a solution for the Euler equations without damping can behave like a centered rarefaction wave, which does not satisfy (1.2) near the vacuum boundary. In fact, $\alpha=1$ in this case. Since the above coordinate transformation works only for $\alpha=2$, the local existence of solutions for $1<\alpha<2$ remains open.

For the systems governing the evolution of the self-gravitating gaseous star, the Euler equation is coupled with the Poisson equation for the gravitational potential determined only by the density distribution of the gas. Under the assumption of spherical symmetry, a family of stationary solutions is found in [17] which satisfy (1.2) near the boundary. In Section 3, we will also apply the coordinate transformation to this system to obtain local existence which includes the stationary solutions.

2. Euler equations. In this section, we will consider the one-dimensional Euler equations for isentropic flow with damping (1.1). For simplicity, we consider the system (1.1) with the following initial and boundary conditions,

$$
\begin{aligned}
& \rho(x, 0)=\rho_{0}(x), \quad u(x, 0)=u_{0}(x) \\
& u(0, t)=0, \quad 0 \leq x \leq a
\end{aligned}
$$

where $a$ is a positive constant, $\rho_{0}(x)>0$ when $0 \leq x<a$ and $\rho_{0}(a)=0$. When the solution is regular, it is easy to see that the vacuum boundary is a particle path. In our case, the vacuum boundary is the particle path through $x=a$.

The characteristics of this system are $\lambda_{1}=u-c, \lambda_{2}=u+c$, and the corresponding Riemann invariants are $r=u-\frac{2}{\gamma-1} c, s=u+\frac{2}{\gamma-1} c$. The equations for $(r, s)$ are

$$
\begin{aligned}
& r_{t}+\lambda_{1} r_{x}=-u, \\
& s_{t}+\lambda_{2} s_{x}=-u,
\end{aligned}
$$

where we normalize the frictional constant $k$ to be 1 .

To illustrate why the classical existence theory for hyperbolic systems fails under the assumption of physical singularity condition (1.2), we consider how the characteristics change near the boundary.

For any point $(\eta, 0), 0 \leq \eta \leq a$, we define the two characteristics lines through $(\eta, 0)$ as

$$
\begin{aligned}
& \frac{\partial x_{i}(\eta, t)}{\partial t}=\lambda_{i}\left(u\left(x_{i}(\eta, t), t\right), c\left(x_{i}(\eta, t), t\right)\right), \\
& x_{i}(\eta, 0)=\eta, \quad i=1,2 .
\end{aligned}
$$

To see how the characteristics change with respect to the initial location $\eta$, we differentiate (2.3) with respect to $\eta$ to yield

$$
\frac{\partial}{\partial t}\left(\frac{\partial x_{i}(\eta, t)}{\partial \eta}\right)=\frac{\partial \lambda_{i}\left(x_{i}(\eta, t), t\right)}{\partial x_{i}} \frac{\partial x_{i}(\eta, t)}{\partial \eta}
$$


i.e.

$$
\frac{\partial x_{i}(\eta, t)}{\partial \eta}=e^{\int_{o l_{\eta}^{i}}^{t} \frac{\partial \lambda_{i}\left(x_{i}(\eta, t), t\right)}{\partial x_{i}} d s}
$$

Here $l_{\eta}^{i}=\left\{(x, t) \mid x=x_{i}(\eta, t)\right\}, i=1,2$, are the characteristic curves passing through $(\eta, 0)$. Since the vacuum boundary in this case is $x=x_{2}(a, t)$, we consider the integral on the right hand side of (2.4) for $x=x_{2}(\eta, t)$ near $x=x_{2}(a, t)$ as follows. Under the assumption (1.2) on the solution, $c\left(x_{2}(\eta, t), t\right)$ behaves like $0(1) \sqrt{x_{2}(a, t)-x_{2}(\eta, t)}$ in a small neighborhood of the vacuum boundary. Therefore, if $u_{x}$ is bounded near the vacuum boundary, we have

$$
\left.\frac{\partial\left(x_{2}(a, t)-x_{2}(\eta, t)\right)}{\partial t}=-0(1) \sqrt{x_{2}(a, t)-x_{2}(\eta, t)}\right),
$$

where $0(1)$ here is a positive quantity. The above equation immediately implies that the characteristics through $\eta$ will meet the vacuum boundary at finite time. If we denote this time by $t_{\eta}$, then

$$
\begin{aligned}
\int_{o l_{\eta}^{2}}^{t_{\eta}} \frac{\partial \lambda_{2}\left(x_{2}(\eta, t), t\right)}{\partial x_{2}} d t & =\int_{o l_{\eta}^{2}}^{t_{\eta}} 0(1)\left(\sqrt{x_{2}(a, t)-x_{2}(\eta, t)}\right)^{-\frac{1}{2}} d t \\
& =0(1) \int_{o l_{\eta}^{2}}^{t_{\eta}}\left(t_{\eta}-t\right)^{-1} d t=\infty
\end{aligned}
$$

This singularity in the integral prevents the application of the classical local existence theory for one-dimensional hyperbolic systems by characteristic method. Furthermore, if we linearize the system in a small neighborhood of a point at the vacuum boundary, then we will have a singular wave equation of the form

$$
u_{t t}-y u_{y y}=L\left(u_{t}, u_{y}, u\right), \quad-\delta \leq t \leq \delta, \quad y \geq 0,
$$

where $L\left(u_{t}, u_{y}, u\right)$ represents the lower order term, $y$ represents the horizontal distance from the vacuum boundary at that point, and $\delta$ is a positive constant. The Cauchy problem for second order hyperbolic equations with degeneracy along a line has been studied by many authors, cf. [20] and reference therein. It has also been studied with the connection to Tricomi problem. However, the degeneracy in (2.6) along the line $y=0$ is different from those in these studies and their local existence theory can not be applied here.

In order to cope with the degeneracy of the characteristics along the vacuum boundary, we will first introduce a coordinate transformation to symmetrize the system and to have non-degenerate propagation speeds. This can be done under the condition (1.2) for physical singularity. Before doing this, it is convenient to rewrite the system (1.1) in the Lagrangian coordinates as follows to fix the vacuum boundary,

$$
\begin{aligned}
& v_{t}-u_{\xi}=0, \\
& u_{t}+p(v)_{\xi}=-u,
\end{aligned}
$$

where $v=\frac{1}{\rho}$ is the specific volume and $\xi=\int_{0}^{x} \rho(y, t) d y$. Without loss of generality, we normalize the total mass $\int_{0}^{a} \rho(x, 0) d x$ to be 1 . Here the pressure function satisfies the $\gamma$-law, i.e., $p(v)=\sigma^{2} v^{-\gamma}, \gamma>1$. Notice that the physical sigularity (1.2) in Eulerian coordinates corresponds to $0<\left|p_{\xi}(v)\right|<\infty$ in the Lagrangian coordinates. 
In the following discussion, both the initial data and the solution satisfy this physical boundary condition. In order to capture this singular behavior of the solution near the vacuum and to symmetrize the system (2.1), we introduce the following coordinate transformation,

$$
1-\xi=y^{\frac{2 \gamma}{\gamma-1}}
$$

Then system (2.1) can be rewritten as

$$
\begin{aligned}
& \phi(v)_{t}-\bar{\mu} u_{y}=0, \\
& u_{t}-\bar{\mu} \phi(v)_{y}=-u,
\end{aligned}
$$

where $\phi(v)=\frac{2 \sqrt{\gamma} \sigma}{\gamma-1} v^{-\frac{\gamma-1}{2}}$, and

$$
\bar{\mu}=\frac{(\gamma-1) \sigma}{\sqrt{\gamma}}\left(v y^{\frac{2}{\gamma-1}}\right)^{-\frac{\gamma+1}{2}}=c\left(y^{-1} \phi\right)^{\frac{\gamma+1}{\gamma-1}}
$$

for some positive constant $c$. Notice that near the vacuum boundary, both $\phi(v)_{y}$ and $\bar{\mu}$ are bounded away from zero as a consequence of (1.2).

In the following, we will apply the fixed point theorem to establish the local existence theorem for the system (2.8) using some energy estimates on the function $\phi(v)$. That is, we will prove the following theorem.

\section{THEOREM 2.1. Under the boundary condition}

$$
u(1, t)=0, \quad \phi(0, t)=0,
$$

if the initial data $(u(y, 0), \phi(y, 0)) \in H^{3}([0,1])$ with $\|u(y, 0)\|_{H^{3}},\|\phi(y, 0)\|_{H^{3}} \leq M_{0}$ for some positive constant $M_{0}$, and $D_{1} \leq y^{-1} \phi(y, 0) \leq D_{2}$ for two positive constants $D_{1}$ and $D_{2}$, then there exists a time $T>0$ such that the system has a unique solution $(u(y, t), \phi(y, t)) \in L^{\infty}\left([0, T], H^{3}([0,1])\right) \cap C\left([0, T], H^{2}([0,1])\right)$ in the region $(y, t) \in$ $[0,1] \times[0, T]$. Futhermore,

$$
D_{1} / 2 \leq y^{-1} \phi(y, t) \leq 2 D_{2}, \quad 0 \leq t \leq T .
$$

Notice here that (2.10) implies that the physical boundary condition (1.2) holds at least in finite time $[0, T]$.

To prove Theorem 2.1, we first reformulate the system (2.9) as

$$
\left(\bar{\mu}^{-1} \phi_{t}\right)_{t}-\left(\bar{\mu} \phi_{y}\right)_{y}+\bar{\mu}^{-1} \phi_{t}=0 .
$$

For this equation, we will prove the following theorem which immediately yields Theorem 2.1. Notice that by the boundary condition at $y=1, u(y, t)$ can be expressed as

$$
u=-\int_{y}^{1} \bar{\mu}^{-1} \phi_{t}(s, t) d s
$$

THEOREM 2.2. Under the hypothesis of Theorem 2.1, there exists a time $T>0$ such that there exists a unique solution

$$
\phi(y, t) \in L^{\infty}\left([0, T], H^{3}([0,1])\right) \cap C\left([0, T], H^{2}([0,1])\right)
$$


satisfying (2.10).

Proof. The proof is based on the fixed point theorem by deriving some energy estimates. We use the following notations.

$$
\left\|\phi_{1}-\phi_{2}\right\| \|_{s}=\sup \left\{\left\|\phi_{1}-\phi_{2}\right\|_{s}: 0 \leq t \leq T\right\},
$$

for some fixed time $T$, where $\|\cdot\|_{s}$ denotes the $H^{s}([0,1])$-norm and $\|\cdot\|=\|\cdot\|_{0}$ for simplicity. When $s=0$, we simplily use $\left\|\left|\phi_{1}-\phi_{2} \|\right|\right.$.

Now we consider the linear equation for $\tilde{\phi}$ with the boundary condition (2.9),

$$
\left(\bar{\mu}^{-1} \tilde{\phi}_{t}\right)_{t}-\left(\bar{\mu} \tilde{\phi}_{y}\right)_{y}+\bar{\mu}^{-1} \tilde{\phi}_{t}=0
$$

where $\bar{\mu}=c\left(y^{-1} \phi\right)^{\frac{\gamma+1}{\gamma-1}}$ defined in (2.8) for a function $\phi$ in the following space:

$$
\begin{aligned}
& X=\left\{\phi(y, t) \mid \phi \in L^{\infty}\left([0, T], H^{3}([0,1])\right) \cap C\left([0, T] ; H^{2}([0,1])\right):\right. \\
& \|\phi\|_{3} \leq c_{0} M_{0}, \quad\left\|\phi\left(t_{1}\right)-\phi\left(t_{0}\right)\right\|_{2} \leq L\left(t_{1}-t_{0}\right), \\
& \left.D_{1} / 2 \leq y^{-1} \phi(y, t) \leq 2 D_{2}, \quad \phi(0, t)=0, \quad \phi_{y}(1, t)=0\right\}
\end{aligned}
$$

where $c_{0}$ is a positive constant defined later. We want to show that $\tilde{\phi} \in X$ and the mapping from $\phi$ to $\tilde{\phi}$ is contractive with respect to the metric defined below for a short time. Notice that the space $X$ can be made into a complete metric space with the metric, cf. [9],

$$
d\left(\phi_{1}, \phi_{2}\right)=\left\||| \phi_{1}-\phi_{2}\right\|_{2} .
$$

We prove for a small time $\tilde{\phi}(y, t) \in X$ first. Multipling (2.12) by $\tilde{\phi}$ and integrating it over $(y, t)$ in $[0,1] \times[0, t]$ yields

$$
\iint\left(\bar{\mu}^{-1} \tilde{\phi}_{t}\right)_{t} \tilde{\phi}-\iint\left(\bar{\mu} \tilde{\phi}_{y}\right)_{y} \tilde{\phi}+\iint \bar{\mu}^{-1} \tilde{\phi}_{t} \tilde{\phi}=0
$$

where, for simplicity, we have used the notation $\iint$ to denote $\int_{0}^{t} \int_{0}^{1} d y d t$. We will also use $\int$ to denote $\int_{0}^{1} d y$ without any ambiguity. By using the boundary condition $\tilde{\phi}(0, t)=0$ and $\tilde{\phi}_{y}(1, t)=0$, we have

$$
\iint\left(-\bar{\mu}^{-1} \tilde{\phi}_{t}^{2}+\bar{\mu} \tilde{\phi}_{y}^{2}\right)+\frac{1}{2} \int \bar{\mu}^{-1} \tilde{\phi}^{2}=-\int \bar{\mu}^{-1} \tilde{\phi} \tilde{\phi}_{t}+\frac{1}{2} \iint\left(\bar{\mu}^{-1}\right)_{t} \tilde{\phi}^{2}+I
$$

where $I$ represents a generic constant depending only on the initial data and $I=0$ if the initial data is zero. Similarly, multipling $(2.12)$ by $\tilde{\phi}_{t}$ and integrating it over $(y, t)$ in $[0,1] \times[0, t]$ gives

$$
\frac{1}{2} \int\left(\bar{\mu}^{-1} \tilde{\phi}_{t}^{2}+\bar{\mu} \tilde{\phi}_{y}^{2}\right)+\iint\left[\bar{\mu}^{-1} \tilde{\phi}_{t}^{2}+\frac{1}{2}\left(\bar{\mu}^{-1}\right)_{t} \tilde{\phi}_{t}^{2}-\frac{1}{2}\left(\bar{\mu}^{-1}\right)_{t} \tilde{\phi}_{y}^{2}\right]=I .
$$

For estimates on higher derivatives, we differentiate (2.12) with respect to $t$ and integrate it by mutiplying $\phi_{t t}$. Then after some calculations, we have

$$
\begin{aligned}
& \frac{1}{2} \int\left[\bar{\mu}^{-1} \tilde{\phi}_{t t}^{2}+\bar{\mu} \tilde{\phi}_{y t}^{2}\right]+\iint\left[\frac{3}{2}\left(\bar{\mu}^{-1}\right)_{t} \tilde{\phi}_{t t}^{2}-\frac{3}{2}(\bar{\mu})_{t} \tilde{\phi}_{y t}^{2}+\bar{\mu}^{-1} \tilde{\phi}_{t t}^{2}\right] \\
= & -\int(\bar{\mu})_{t} \tilde{\phi}_{y t} \tilde{\phi}_{y}+\iint\left[(\bar{\mu})_{t t} \tilde{\phi}_{y t} \tilde{\phi}_{y}-\left(\bar{\mu}^{-1}\right)_{t t} \tilde{\phi}_{t t} \tilde{\phi}_{t}\right]+I .
\end{aligned}
$$


Notice that $\bar{\mu}_{t}=\bar{\mu}^{\prime} y^{-1} \phi_{t}$ which is bounded because $\phi(0, t)=0$ and $\phi \in X$. Here we use ' to denote the differentiation with respect to the variable $y^{-1} \phi$. As for $\left(\bar{\mu}^{-1}\right)_{t t}$, we have

$$
\left(\bar{\mu}^{-1}\right)_{t t}=\left(\bar{\mu}^{-1}\right)^{\prime} y^{-1} \phi_{t t}+\left(\bar{\mu}^{-1}\right)^{\prime \prime} y^{-2} \phi_{t}^{2} .
$$

Noticing that $\phi_{t t}$ and $y^{-1} \phi_{t}$ are bounded, the right hand side of (2.15) is bounded by

$$
\begin{aligned}
& 0(1) \iint\left[\tilde{\phi}_{t t}^{2}+\tilde{\phi}_{y t}^{2}+y^{-2}\left(\tilde{\phi}_{t}^{2}+\tilde{\phi}_{y}^{2}\right)\right]+\epsilon \int \tilde{\phi}_{y t}^{2}+0(1) \epsilon^{-1} \int \tilde{\phi}_{y}^{2}+I \\
\leq & 0(1) \iint\left(\tilde{\phi}_{t t}^{2}+\tilde{\phi}_{y t}^{2}+\tilde{\phi}_{y y}^{2}\right)+\epsilon \int \tilde{\phi}_{y t}^{2}+0(1) \epsilon^{-1} \int\left(\tilde{\phi}_{y}^{2}+\tilde{\phi}_{t}^{2}\right)+I,
\end{aligned}
$$

where $\epsilon$ is a small positive constant and we have used

$$
\begin{aligned}
\int y^{-2} \phi_{t}^{2} & =-\left.y^{-1} \phi_{t}^{2}\right|_{0} ^{1}+2 \int y^{-1} \phi_{t} \phi_{y t} \\
& \leq-\phi(1, t)_{t}^{2}+\frac{1}{2} \int y^{-2} \phi_{t}^{2}+8 \int \phi_{y t}^{2}
\end{aligned}
$$

and

$$
\begin{aligned}
\int y^{-2} \phi_{y}^{2} & =-\left.y^{-1} \phi_{y}^{2}\right|_{0} ^{1}+2 \int y^{-1} \phi_{y} \phi_{y y} \\
& \leq \frac{1}{2} \int y^{-2} \phi_{y}^{2}+8 \int \phi_{y y}^{2} .
\end{aligned}
$$

Now we differentiate (2.12) with respect to $t$ twice and integrate it by multiplying $\phi_{t t t}$. Then we have

$$
\begin{aligned}
& \iint\left[\left(\bar{\mu}^{-1}\right)_{t t t} \tilde{\phi}_{t} \tilde{\phi}_{t t t}+3\left(\bar{\mu}^{-1}\right)_{t t} \tilde{\phi}_{t t} \tilde{\phi}_{t t t}+3\left(\bar{\mu}^{-1}\right)_{t} \tilde{\phi}_{t t t}^{2}\right. \\
& \left.+\bar{\mu}^{-1}\left(\frac{\tilde{\phi}_{t t t}^{2}}{2}\right)_{t}+\tilde{\phi}_{y t t t}\left(\bar{\mu} \tilde{\phi}_{y}\right)_{t t}+\left(\bar{\mu}^{-1} \tilde{\phi}_{t}\right)_{t t} \tilde{\phi}_{t t t}\right]=0
\end{aligned}
$$

The last two terms in (2.16) can be estimated as follows.

$$
\begin{aligned}
\iint \tilde{\phi}_{y t t t}\left(\bar{\mu} \tilde{\phi}_{y}\right)_{t t}= & \int\left[(\bar{\mu})_{t t} \tilde{\phi}_{y} \tilde{\phi}_{y t t}+2 \bar{\mu}_{t} \tilde{\phi}_{y t} \tilde{\phi}_{y t t}+\bar{\mu} \tilde{\phi}_{y t t}^{2}\right] \\
& -\iint\left[(\bar{\mu})_{t t t} \tilde{\phi}_{y} \tilde{\phi}_{y t t}+3(\bar{\mu})_{t t} \tilde{\phi}_{y t} \tilde{\phi}_{y t t}+3 \bar{\mu}_{t} \tilde{\phi}_{y t t}^{2}+\bar{\mu}\left(\frac{\phi_{y t t}^{2}}{2}\right)_{t}\right] \\
= & \int\left[\frac{1}{2} \bar{\mu} \tilde{\phi}_{y t t}^{2}+(\bar{\mu})_{t t} \tilde{\phi}_{t} \tilde{\phi}_{y t t}+2(\bar{\mu})_{t} \tilde{\phi}_{y t} \tilde{\phi}_{y t t}\right] \\
& -\iint\left[\bar{\mu}_{t t t} \tilde{\phi}_{y} \tilde{\phi}_{y t t}+3 \bar{\mu}_{t t} \tilde{\phi}_{y t} \tilde{\phi}_{y t t}\right]+I
\end{aligned}
$$

And

$$
\iint\left(\bar{\mu}^{-1} \tilde{\phi}_{t}\right)_{t t} \tilde{\phi}_{t t t}=\iint\left[\bar{\mu}^{-1} \tilde{\phi}_{t t t}^{2}+\left(\bar{\mu}^{-1}\right)_{t t} \tilde{\phi}_{t} \tilde{\phi}_{t t t}+2\left(\bar{\mu}^{-1}\right)_{t} \tilde{\phi}_{t t} \tilde{\phi}_{t t t}\right]
$$


Thus (2.16) yields

$$
\begin{aligned}
& \frac{1}{2} \int\left(\bar{\mu}^{-1} \tilde{\phi}_{t t t}^{2}+\bar{\mu} \tilde{\phi}_{y t t}^{2}\right)+\iint \bar{\mu}^{-1} \tilde{\phi}_{t t t}^{2} \\
= & -\iint\left[\left(\bar{\mu}^{-1}\right)_{t t t} \tilde{\phi}_{t} \tilde{\phi}_{t t t}-3\left(\bar{\mu}^{-1}\right)_{t t} \tilde{\phi}_{t t} \tilde{\phi}_{t t t}+\bar{\mu}_{t t t} \tilde{\phi}_{y} \tilde{\phi}_{y t t}+3 \bar{\mu}_{t t} \tilde{\phi}_{y t} \tilde{\phi}_{y t t}\right. \\
& \left.-\bar{\mu}_{t t} \tilde{\phi}_{t} \tilde{\phi}_{t t t}-2\left(\bar{\mu}^{-1}\right)_{t} \tilde{\phi}_{t t} \tilde{\phi}_{t t t}\right]-\int\left[\bar{\mu}_{t t} \tilde{\phi}_{y} \tilde{\phi}_{y t t}-2 \bar{\mu}_{t} \tilde{\phi}_{y t} \tilde{\phi}_{y t t}\right]+I .
\end{aligned}
$$

The terms in (2.17) can be estmated as before except for the terms involving $\left(\bar{\mu}^{-1}\right)_{t t t}$ or $\bar{\mu}_{t t t}$, which contains the terms $y^{-3} \phi_{t}^{3}, y^{-2} \phi_{t} \phi_{t t}$ and $y^{-1} \phi_{t t t} . y^{-3} \phi_{t}^{3}$ is bounded because $\phi \in X$ and the terms with $y^{-2} \phi_{t} \phi_{t t}$ can be discussed as in (2.15). It remains to estimate the terms with $y^{-1} \phi_{t t t}$. That is, we need to estimate $\iint y^{-1} \phi_{t t t}\left(\tilde{\phi}_{t} \tilde{\phi}_{t t t}+\right.$ $\left.\tilde{\phi}_{y} \tilde{\phi}_{y t t}\right)$ as follows.

$$
\begin{aligned}
& \iint y^{-1} \phi_{t t t}\left(\tilde{\phi}_{t} \tilde{\phi}_{t t t}+\tilde{\phi}_{y} \tilde{\phi}_{y t t}\right) \\
\leq & \frac{1}{4} \iint \phi_{t t t}^{2}+\iint\left(y^{-2} \tilde{\phi}_{t}^{2}+y^{-1}\left|\tilde{\phi}_{y}\right|\right)\left(\tilde{\phi}_{t t t}^{2}+\tilde{\phi}_{y t t}^{2}\right) \\
\leq & \frac{1}{4} \iint \phi_{t t t}^{2}+\int_{0}^{t}\left(\left|y^{-1} \tilde{\phi}_{t}\right|_{\infty}^{2}+y^{-1}\left|\tilde{\phi}_{y}\right|_{\infty}\right) \int\left(\tilde{\phi}_{t t t}^{2}+\tilde{\phi}_{y t t}^{2}\right) \\
\leq & \frac{1}{4} \iint \phi_{t t t}^{2}+C \int_{0}^{t}\|\tilde{\phi}\|_{3}^{4},
\end{aligned}
$$

for some constant $C$, where we have used the boundary condition $\tilde{\phi}_{y}(1, t)=0$.

Combining (2.13), (2.14), (2.15) and (2.18) and using the equation (2.12), we have the estimate

$$
\|\tilde{\phi}\|_{3}^{2}(t) \leq C \int_{0}^{t}\left[\|\tilde{\phi}\|_{3}^{2}(s)+\|\tilde{\phi}\|_{3}^{4}(s)\right] d s+\frac{c_{0}^{2}}{4}\left\|\phi_{0}\right\|_{3}^{2}+\frac{c_{0}^{2} M_{0}^{2}}{4},
$$

for some positive constants $C$ and $c_{0}$. Here we have used the fact that $\phi \in X$. (2.19) immediately gives

$$
\|\tilde{\phi}\|_{3}^{2} \leq \frac{c_{0}^{2} M_{0}^{2} e^{C T}}{c_{0}^{2} M_{0}^{2}\left(1-e^{C T}\right)+2} .
$$

Therefore, there exists a small time $T>0$ such that

$$
\|\tilde{\phi}\|_{3} \leq c_{0} M_{0}
$$

By using the equation and the above estimates, it is easy to see that $\frac{d}{d t}\|\tilde{\phi}\|_{2} \leq L$ for some constant $L$. Since $\phi_{y t}$ is bounded, (2.10) holds for $T>0$ being sufficiently small. Hence the function $\tilde{\phi}$ is also in the space $X$.

Now we turn to prove that the mapping $F$ from $\phi$ to $\tilde{\phi}$ is contractive with respect to the metric $d$. Let $F$ map two functions $\phi_{i}$ in $X$ to two functions $\tilde{\phi}_{i} \in X$ respectively, $i=1,2$. Let $\delta g=g_{1}-g_{2}$ for any function $g$, and $\bar{\mu}_{i}=c\left(y^{-1} \phi_{i}\right)^{\frac{\gamma+1}{\gamma-1}}, i=1,2$. Then the equation for $\delta \tilde{\phi}$ is

$$
\begin{aligned}
& \left(\bar{\mu}_{1}^{-1}(\delta \tilde{\phi})_{t}\right)_{t}-\left(\bar{\mu}_{1}(\delta \tilde{\phi})_{y}\right)_{y}+\bar{\mu}_{1}^{-1}(\delta \tilde{\phi})_{t} \\
= & \left(\left(\delta \bar{\mu}^{-1}\right) \tilde{\phi}_{1 t}\right)_{t}-\left((\delta \bar{\mu}) \tilde{\phi}_{1 y}\right)_{y}+(\delta \bar{\mu})^{-1} \tilde{\phi}_{1 t} .
\end{aligned}
$$


By multipling $(2.20)$ by $\delta \tilde{\phi}+\delta \tilde{\phi}_{t}$ and integrating over the region $[0,1] \times[0, t]$, after some calculations we have

$$
\|\delta \tilde{\phi}\|_{1}^{2} \leq C \int_{0}^{t}\left[\|\delta \tilde{\phi}\|_{1}^{2}+\|\delta \phi\|_{1}^{2}\right] d s,
$$

where the constant $C$ depending on $\left\|\left|\tilde{\phi}_{i}\right|\right\|_{3}$ and $\left\|\left|\phi_{i}\right|\right\|_{3}, i=1,2$.

By differentiating (2.20) with respect to $t$ and multiplying it by $\delta \tilde{\phi}_{t t}$, then the integration over the region $[0,1] \times[0, t]$ and combining with $(2.21)$ give

$$
\|\delta \tilde{\phi}\|_{2}^{2} \leq C \int_{0}^{t}\left[\|\delta \tilde{\phi}\|_{2}^{2}+\|\delta \phi\|_{2}^{2}\right] d s .
$$

Thus for small time, still denoted by $T$, we have from (2.22) that

$$
d\left(\tilde{\phi}_{1}, \tilde{\phi}_{2}\right) \leq \Lambda d\left(\phi_{1}, \phi_{2}\right)
$$

for some positive constant $\Lambda<1$.

Therefore, $F$ is a contraction on $X$ and so has a fixed point $\phi(y, t) \in X$. This completes the proof of Theorem 2.2 .

3. Euler-Poisson equations. In this section, we will apply the same coordinate tranformation to another physical system for the evolution of gaseous stars under the assumption of spherical symmetry. The local existence of solutions including the stationary solutions will be given. The Euler-Poisson equations in $\mathbf{R}^{\mathbf{3}}$ can be written as

$$
\begin{aligned}
& \tilde{\rho}_{t}+\nabla \cdot(\tilde{\rho} \vec{u})=0, \\
& \tilde{\rho} \vec{u}_{t}+\tilde{\rho} \vec{u} \cdot \nabla \vec{u}+\nabla p(\tilde{\rho})+\tilde{\rho} \nabla \Phi=0, \\
& \Delta \Phi=4 \pi \tilde{\rho},
\end{aligned}
$$

where $\tilde{\rho}$ is the density, $\vec{u}$ is the velocity, $p(\tilde{\rho})=\sigma^{2} \tilde{\rho}^{\gamma}$ with $\gamma>1$ is the pressure, and $\Phi$ is the gravitational potential. Under the assumption of spherical symmetry, (3.1) is reduced to

$$
\begin{aligned}
& \tilde{\rho}_{t}+u \tilde{\rho}_{r}+\tilde{\rho} u_{r}+\frac{2}{r} \tilde{\rho} u=0, \\
& \tilde{\rho}\left(u_{t}+u u_{r}\right)+p(\tilde{\rho})_{r}+\tilde{\rho} \Phi_{r}=0 \\
& \left(r^{2} \Phi_{r}\right)_{r}=4 \pi r^{2} \tilde{\rho}
\end{aligned}
$$

where $r=|x|$ is the radius.

When $\frac{6}{5}<\gamma<2$, there exist stationary solutions with spherical symmetry in the form of, cf. [17],

$$
\rho=\left(\frac{\sigma^{2} A^{2} \gamma}{4 \pi(\gamma-1)}\right)^{\frac{1}{2-\gamma}} \theta(A|x|)^{\frac{1}{\gamma-1}}, \quad u=0
$$

where $A$ is an arbitrary positive constant and $\theta(r)$ is the "Lane-Emden function" of index $\frac{1}{\gamma-1}$ satisfying, cf. [4],

$$
\frac{d^{2}}{d r^{2}} \theta+\frac{2}{r} \frac{d}{d r} \theta+\theta^{\frac{1}{\gamma-1}}=0, \quad \theta(0)=1, \quad \frac{d}{d r} \theta(0)=0 .
$$


Furthermore, there exists $R=R_{\gamma}$ such that $\theta(r)>0$ when $0 \leq r<R$ and $\theta(R)=0$. And the solutions behaves like

$$
\rho(r)=C(R-r)^{\frac{1}{\gamma-1}}\left[1+P\left(R-r,(R-r)^{\frac{\gamma}{\gamma-1}}\right)\right],
$$

when $r \rightarrow R-0$. Here $C$ is a positive constant and $P$ is a double power series with positive radii of convergence. Notice that this behavior is precisely the one stated in (1.2). The local existence result in [17] does not include this family of stationary solutions. For the rest of this paper, we will prove a local existence result which includes the stationary solutions outside a fixed ball. That is, we consider the system (3.2) when $r \geq r_{0}$ and $u\left(r_{0}, t\right)=0$. Here $r_{0}<R_{\gamma}$ is a positive constant.

First we also rewrite the system (3.2) in the Lagrangian coordinates. Let

$$
\rho=r^{2} \tilde{\rho}, \quad x=\int_{r_{0}}^{r} \rho(s, t) d s, \quad r \geq r_{0} .
$$

By normalize the total mass $\int_{r_{0}}^{\infty} \rho(s, t) d s$ to be 1 , the system (3.2) can be written as

$$
\begin{aligned}
& \rho_{t}+\rho^{2} u_{x}=0, \\
& u_{t}+\frac{\sigma^{2} \gamma \rho_{x}}{\rho^{1-\gamma} r^{2 \gamma-2}}=\frac{2 \sigma^{2} \gamma \rho^{\gamma-1}}{r^{2 \gamma-1}}-\Phi_{r}, \\
& \left(r^{2} \Phi_{r}\right)_{r}=4 \pi \rho .
\end{aligned}
$$

We will consider the system with the following initial boundary conditions:

$$
\begin{aligned}
& u(x, 0)=u_{0}(x), \quad \rho(x, 0)=\rho_{0}(x), \quad 0<x<1, \\
& u(0, t)=0, \quad \rho(1, t)=0, \quad \Phi_{r}(0, t)=\Phi_{0},
\end{aligned}
$$

where $\Phi_{0}$ is a constant. The boundary condition $u(0, t)=0$ implies that

$$
\frac{\sigma^{2} \gamma \rho_{x}}{\rho^{1-\gamma} r_{0}^{2 \gamma-2}}-\left.\frac{2 \sigma^{2} \gamma \rho^{\gamma-1}}{r_{0}^{2 \gamma-1}}\right|_{x=0}=\Phi_{0}
$$

As for the Euler equations with damping, we first introduce the coordinate tranformation $1-x=y^{\frac{2 \gamma}{\gamma-1}}$. Under this transformation, the system (3.3) can be written as

$$
\begin{aligned}
& \phi_{t}-\bar{\mu} u_{y}=0 \\
& u_{t}-\frac{c_{1}}{r^{2 \gamma-2}} \bar{\mu} \phi_{y}=\frac{c_{2}}{r^{2 \gamma-1}} \phi^{2}-\frac{4 \pi}{r^{2}}\left[1-y^{\frac{2 \gamma}{\gamma-1}}+\frac{r_{0}^{2} \Phi_{0}}{4 \pi}\right]
\end{aligned}
$$

where

$$
\phi(v)=\int_{v}^{\infty} \mu(s) d s, \quad \mu(v)=\sigma \sqrt{\gamma} v^{-\frac{\gamma+1}{2}}, \quad v=\rho^{-1}
$$

and $\bar{\mu}=c\left(y^{-1} \phi\right)^{\frac{\gamma+1}{\gamma-1}}$, and $c_{i}$ are positive constants, $i=1,2$. For the system (3.5), we will prove the following theorem.

THEOREM 3.1. Under the boundary condition

$$
u(1, t)=0, \quad \phi(0, t)=0
$$


if the initial data $(u(y, 0), \phi(y, 0)) \in H^{3}([0,1])$ with $\|u(y, 0)\|_{H^{3}},\|\phi(y, 0)\|_{H^{3}} \leq M_{0}$ for some positive constant $M_{0}$, and $D_{1} \leq y^{-1} \phi(y, 0) \leq D_{2}$ for two positive constants $D_{1}$ and $D_{2}$, then there exists a time $T>0$ such that the system has a unique solution $(u(y, t), \phi(y, t)) \in L^{\infty}\left([0, T], H^{3}([0,1])\right) \cap C\left([0, T], H^{2}([0,1])\right)$ in the region $(y, t) \in$ $[0,1] \times[0, T]$. Futhermore,

$$
D_{1} / 2 \leq y^{-1} \phi(y, t) \leq 2 D_{2}, \quad 0 \leq t \leq T .
$$

(3.6) implies that the physical boundary condition (1.2) holds at least in finite time $[0, T]$.

For convenience, we also rewrite the system (3.3) into a scalar second order differential equation for $\phi$,

$$
\left(\bar{\mu}^{-1} \phi_{t}\right)_{t}-\left(\frac{c_{1}}{r^{2 \gamma-2}} \bar{\mu} \phi_{y}\right)_{y}=\left(\frac{c_{2}}{r^{2 \gamma-1}} \phi^{2}\right)_{y}-\left(\frac{r_{0}^{2} \Phi_{0}}{r^{2}}\right)_{y}+\left(\frac{4 \pi}{r^{2}}\left(y^{\frac{2 \gamma}{\gamma-1}}-1\right)\right)_{y},
$$

and $u$ can also be defined as

$$
u(y, t)=-\int_{y}^{1} \bar{\mu}^{-1} \phi_{t}(s, t) d s,
$$

by using the boundary condition on $y=1$. For this equation, the corresponding theorem to Theorem 3.1 can be stated as follows.

THEOREM 3.2. Under the hypothesis of Theorem 3.1, there is a time $T>0$ such that there exists a unique solution $\phi(y, t) \in L^{\infty}\left([0, T], H^{3}([0,1])\right) \cap C\left([0, T], H^{2}([0,1])\right)$ satisfying (3.6).

Proof. The proof is also based on the fixed point theorem by deriving some energy estimates on the corresponding linear equation. For a small fixed time $T$ to be determined later, we consider the function space,

$$
\begin{aligned}
& X=\left\{\phi(y, t) \mid \phi \in L^{\infty}\left([0, T], H^{3}([0,1])\right) \cap C\left([0, T] ; H^{2}([0,1])\right):\right. \\
& \|\phi\|_{3} \leq c_{0} M_{0}, \quad\left\|\phi\left(t_{1}\right)-\phi\left(t_{0}\right)\right\|_{2} \leq L\left(t_{1}-t_{0}\right), \quad D_{1} / 2 \leq y^{-1} \phi(y, t) \leq 2 D_{2}, \\
& \left.\phi(0, t)=0, \quad \frac{c_{1} c}{r_{0}^{2 \gamma-2}} \phi^{\frac{\gamma-1}{\gamma-1}} \phi_{y}+\left.\frac{c_{2}}{r_{0}^{2 \gamma-1}} \phi^{2}\right|_{y=1}=\Phi_{0}\right\},
\end{aligned}
$$

where $c_{0}$ will be defined later. With the metric $d\left(\phi_{1}, \phi_{2}\right)=\left\||| \phi_{1}-\phi_{2} \mid\right\|_{2}$, the space $X$ with $d$ is a complete metric space.

We are going to show that the mapping $F$ from $\phi \in X$ to $\tilde{\phi}$ given by the following linear equation is a contraction on $X$ :

$$
\left(\bar{\mu}^{-1} \tilde{\phi}_{t}\right)_{t}-\left(\frac{c_{1}}{r^{2 \gamma-2}} \bar{\mu} \tilde{\phi}_{y}\right)_{y}=\left(\frac{c_{2}}{r^{2 \gamma-1}} \phi^{2}\right)_{y}-\left(\frac{r_{0}^{2} \Phi_{0}}{r^{2}}\right)_{y}+\left(\frac{4 \pi}{r^{2}}\left(y^{\frac{2 \gamma}{\gamma-1}}-1\right)\right)_{y},
$$

with the boundary condition

$$
\tilde{\phi}(0, t)=0, \quad \frac{c_{1} c}{r_{0}^{2 \gamma-2}} \tilde{\phi}^{\frac{\gamma-1}{\gamma-1}} \tilde{\phi}_{y}+\left.\frac{c_{2}}{r_{0}^{2 \gamma-1}} \tilde{\phi}^{2}\right|_{y=1}=\Phi_{0} .
$$

Here $\bar{\mu}$ and $r$ in (3.8) are defined by $\phi$. First we can also apply energy method to prove that $\tilde{\phi} \in X$. The proof is similar to the one in Section 2 for Euler equations with damping except the discussion on the extra terms on the right hand side, the 
non-homogeneous boundary condition on $y=1$ and the differentiation on the radius $r$. We omit the details of the proof by giving the main difference between the proof here from the one in Section 2.

Since

$$
r=r_{0}+\int_{0}^{x} v(s, t) d s
$$

we have

$$
\begin{aligned}
& r_{t}=\int_{0}^{x} v_{t}(s, t) d s=0(1) \int_{0}^{y} \phi^{-\frac{\gamma+1}{\gamma-1}} \phi_{t} x_{y} d y=0(1) \int_{0}^{y} \bar{\mu}^{-1} \phi_{t} d y \\
& r_{y}=v x_{y}=0(1) \phi^{-\frac{2}{\gamma-1}} y^{\frac{\gamma+1}{\gamma-1}} \\
& r_{y t}=0(1) y^{\frac{\gamma+1}{\gamma-1}} \phi^{-\frac{\gamma+1}{\gamma-1}} \phi_{t}=0(1) \bar{\mu}^{-1} \phi_{t} \\
& y_{y t t}=0(1)\left(\bar{\mu}^{-1}\right)_{t} \phi_{t}+0(1) \bar{\mu}^{-1} \phi_{t t}
\end{aligned}
$$

which are all bounded because $\phi \in X$.

Now we discuss the boundary terms which come from the integration by parts at $y=1$. It can be seen that it is equivalent to estimating the following

$$
\left.\int_{0}^{t}\left[\left(\frac{1}{r^{2 \gamma-2}} \bar{\mu} \tilde{\phi}_{y}\right)\left(\tilde{\phi}+\tilde{\phi}_{t}\right)+\left(\frac{1}{r^{2 \gamma-2}} \bar{\mu} \tilde{\phi}_{y}\right)_{t} \phi_{t t}+\left(\frac{1}{r^{2 \gamma-2}} \bar{\mu} \tilde{\phi}_{y}\right)_{t t} \tilde{\phi}_{t t t}\right]\right|_{y=1} d s
$$

Notice that at $y=1$, the boundary condition and the equation (3.8) imply that both $\tilde{\phi}_{t t t}$ and $\tilde{\phi}_{y t t}$ can be expressed in terms of $\phi$ and $\tilde{\phi}$ and their derivatives with order less than 2 with bounded coefficients due to (3.9). Therefore, the above error terms at the boundary $y=1$ are bounded by

$$
\begin{aligned}
& 0(1) \tilde{\phi}_{t t}^{2}(1, t)+0(1) \int_{0}^{t}\left[|\tilde{\phi}|^{2}+\left|\tilde{\phi}_{t}\right|^{2}+\left|\tilde{\phi}_{y}\right|^{2}+\left|\tilde{\phi}_{t t}\right|^{2}+\left|\tilde{\phi}_{y t}\right|^{2}+\left|\tilde{\phi}_{y y}\right|^{2}\right] d s \\
\leq & C \epsilon^{-1} \int \tilde{\phi}_{t t}^{2}+\epsilon \int \tilde{\phi}_{t t t}^{2}+C \iint\|\tilde{\phi}\|_{3}^{2},
\end{aligned}
$$

where $\epsilon$ is a small constant.

Now the energy method as in Section 2 can be applied with the estimates (3.9) and (3.10) and it gives the estimate

$$
\|\tilde{\phi}\|_{3}^{2}(t) \leq C \int_{0}^{t}\left[\|\tilde{\phi}\|_{3}^{2}(s)+\|\tilde{\phi}\|_{3}^{4}(s)\right] d s+\frac{c_{0}^{2}}{4}\left\|\phi_{0}\right\|_{3}^{2}+\frac{c_{0}^{2} M_{0}^{2}}{4}
$$

for some positive constants $C$ and $c_{0}$. (3.11) immediately implies that $\|\tilde{\phi}\| \|_{3} \leq c_{0} M_{0}$ for a short time $T>0$. The other estimates for $\tilde{\phi}$ can be obtained immediately so that $\tilde{\phi} \in X$.

Now we turn to show that the mapping $F$ is contractive in the metric $d$ on $X$. We denote the right hand side of (3.8) by $G(\phi, y)$. Let $F$ map two functions $\phi_{i}$ in $X$ to two functions $\tilde{\phi}_{i} \in X$ respectively, $i=1,2$. Following the notations in Section 2, the equation for $\delta \tilde{\phi}$ is

$$
\left(\bar{\mu}_{1}^{-1}(\delta \tilde{\phi})_{t}\right)_{t}-\left(\frac{c_{1}}{r^{2 \gamma-2}} \bar{\mu}_{1}(\delta \tilde{\phi})_{y}\right)_{y}=\delta G+\left(\left(\delta \bar{\mu}^{-1}\right) \tilde{\phi}_{1 t}\right)_{t}-\left(\left(\delta\left(\frac{c_{1}}{r^{2 \gamma-2}} \dot{\bar{\mu}}\right)\right) \tilde{\phi}_{1 y}\right)_{y} .
$$


By multipling (3.12) by $\delta \tilde{\phi}+\delta \tilde{\phi}_{t}$ and integrating over the region $[0,1] \times[0, t]$, after some calculations, we have

$$
\|\delta \tilde{\phi}\|_{1}^{2} \leq C \int_{0}^{t}\left[\|\delta \tilde{\phi}\|_{1}^{2}+\|\delta \phi\|_{1}^{2}\right] d s
$$

where the constant $C$ depends on $\left\|\left|\tilde{\phi}_{i}\right|\right\|_{3}$ and $\left\|\left|\phi_{i}\right|\right\|_{3}, i=1,2$.

By differentiating (3.12) with respect to $t$ and multiplying it by $\delta \tilde{\phi}_{t t}$ and then integrating over the region $[0,1] \times[0, t]$, we have from $(3.13)$,

$$
\|\delta \tilde{\phi}\|_{2}^{2} \leq C \int_{0}^{t}\left[\|\delta \tilde{\phi}\|_{2}^{2}+\|\delta \phi\|_{2}^{2}\right] d s .
$$

Thus for small time, still denoted by $T>0$, we have

$$
d\left(\tilde{\phi}_{1}, \tilde{\phi}_{2}\right) \leq \Lambda d\left(\phi_{1}, \phi_{2}\right)
$$

for some positive constant $\Lambda<1$.

Therefore the mapping $F$ is a contraction on $X$ and there is a fixed point $\phi(y, t) \in$ $X$ which is the solution to the system (3.7). This completes the proof of the theorem.

REMARK 3.1. The space of $\phi$ and $u$ for both the systems of Euler equations with damping and Euler-Possion equations can be $H^{3+s}([0,1])$ for any non-negative integer $s$. The proof for the higher derivatives in this case will be similar to the estimates in $H^{3}([0,1])$.

REMARK 3.2. When we consider the solutions which is a small perturbation of the family of special solutions in [11] for Euler equations with damping or the stationary solutions to the Euler-Possion equations with spherical symmetry, solutions for larger time interval can be obtained by deriving the a priori estimates on the solution and using the above local existence theorems.

\section{REFERENCES}

[1] D. G. Aronson, L. A. Caffarelli, and S. Kamin, How an initial stationary interface begins to move in porous media flow, SIAM J. Math., Anal., 14 (1983), pp. 639-658.

[2] G. J. BARENBLATT, On one class of solutions of the one-dimensional problem of non-stationary filtration of a gas in a porous medium, Prikl. Mat. i Mekh., 17 (1953), pp. 739-742.

[3] M. BEZARD, Existence locale pour les equations d'Euler-Possion, Japan J. Indust. Appl. Math., 10 (1993), pp. 431-450.

[4] S. Chandrasekhar, An Introduction to the Study of Steller Structure, University of Chicago Press, 1938.

[5] C.M. DAfermos, Polygonal approximations of solutions of the initial value problem for a conservation law, J. Math. Anal. Appl., 38 (1972), pp. 33-41.

[6] P. Gamblin, Solution reguliere a temps petit pour l'equation d'Euler-Possion, Comm. Partial Diff. Equations, 18 (1993), pp. 731-745.

[7] M. Grassin AND D. SerRe, Global smooth solutions to Euler equations for an isentropic perfect gas, C.R. Acad. Sci. Paris Sér. I Math, 325:7 (1997), pp. 721-726.

[8] L. HSIAO AND T.-P. LIU, Convergence to nonlinear diffusion waves for solutions of a system of hyperbolic conservation laws with damping, Comm. Math. Phys., 143 (1992), pp. 599-605.

[9] T. KATO, The Cauchy problem for quasi-linear symmetric hyperbolic systems, Arch. Rational. Mech. Anay., 58 (1945), pp. 181-205.

[10] L. W. LiN, On the vacuum state for the equations of isentropic gas dynamics, J. of Math. Anal. Appl., 121:2 (1987), pp. 406-425. 
[11] T.-P. LIU, Compressible flow with damping and vacuum, Japan J. Appl. Math., 13:1 (1996), pp. 25-32.

[12] T.-P. LIU, Z.P. XIN, AND T. YANG, Vacuum states for compressible flow, Discrete and Continuous Dynamical Systems, 4:1 (1998), pp. 1-32.

[13] T.-P. LIU AND T. YANG, Compressible Euler equations with vacuum, Journal of Differential Equations, 140:2 (1997), pp. 223-237.

[14] T.-P. LIU AND J. SMOLLER, On the vacuum state for isentropic gas dynamics equations, Advances in Math., 1 (1980), pp. 345-359.

[15] T. MaKino, Blowing up solutions of the Euler-Poisson equation for the evolution of gaseous stars, Transport Theory and Statistical Physics, 21 (1992), pp. 615-624.

[16] T. Makino et B. Perthame, Sur les solutions à symétric sphérique de léquation d'EulerPoisson pour lévolution d'etoiles gazeuses, Japan J. Appl. Math., 7 (1990), pp. 165-170.

[17] T. MAKINo, On a local existence theorem for the evolution equations of gaseous stars, in Pattern and Waves, North-Holland, 1986, pp. 459-479.

[18] T. Makino, S. UKaI, AND S. Kawashima, Sur la solution à support compact de l'équation d'Euler compressible, Japan J. Appl. Math., 3 (1986), pp. 249-257.

[19] T. NishidA, Nonlinear hyperbolic equations and related topics in fluid dynamics, Publ. Math. D'Orsay, No. 78-02, 1978, pp. 46-53.

[20] O.A. Oleinik, On the Cauchy problem for weakly hyperbolic equations, Comm. Pure Appl. Math., XXIII (1970), pp. 569-586.

[21] D. SERRE, Sur l'equation mondimensionnelle d'un fluide visqueux, compressible et conducteur de chaleur, Comptes rendus Acad. des Sciences, 303(1986), pp. 703-706.

[22] T. Sideris, Formation of singularities in three-dimensional compressible fluids, Comm. Math. Phys., 101 (1985), pp. 475-485.

[23] Z. XIN, Blow-up of smooth solutions to the compressible Navier-Stokes equations with compact density, Comm. Pure Appl. math., 51 (1998), pp. 229-240. 
